\title{
Posture and depth adjustable 3D body model for
}

\author{
individual pattern making
}

\begin{abstract}
Purpose; This paper describes the development of an interactive body model that can be altered to match individual body perimeter, postures and depth for the purpose of computerized pattern making.
\end{abstract}

Methodology/Approach; Construction of the posture and depth adjustable body model requires the extraction of ten points, adjustment of coordinate points, linking of points by spline curves, control of section lengths and selectability of three hip types. Front to back depth of the model is adjusted by scaling ratio.

Findings; We achieved good results in modelling back shapes, such as flat shape and stoop shape, and of modelling various hip shapes, such as flat shape and protruding shape. Also our presented body model is able to accurately simulate individual depth of bust, waist and hips. Silhouette comparison between the fully adjusted virtual body model and real body shapes shows an almost perfect match. We developed a primary dialog for altering perimeter, length and depth, and a posture dialog for controlling back and hip shapes.

Originality/value; By making fine adjustments to posture and depth, it is possible to make patterns which result in clothing that not only fits well, but also exhibits other desirable properties. This system could, therefore, be seen as a major step forward in pattern making.

Keyword: 3D body measurement, Body model, Pattern making, Posture, Depth

\section{Introduction}

As peoples' awareness of quality and functionality grows, so does their demand for durable and reliable products. In particular, consumers with great purchasing power are attempting to express their personality by means of individual product choice. Manufacturers are faced with an uninterrupted trend towards individualization (Hashizume S. and Nagata M., 1999; Watanabe Y., 1999). However many women still have difficulty in finding stylish and comfortably fitting garments. For example, many are dissatisfied with the fit around the neckline and shoulders, and badly balanced hemlines of jackets and skirts. Problems of fit relate to individual characteristics of body shapes (Takabu H. and Matsuyama Y., 1987; Masuda T. and Imaoka H., 2002; Matsuyama Y., Yamazaki I. and Watanabe K., 1998). In this study, 'individual characteristics' means posture difference; especially back shapes and hip shapes.

Variations in individual's back shapes and hip shapes are major obstacle in pattern making. For 
example, there are flat back shapes, stooped back shapes, and flat hip shapes and protruding hip shapes. Hem lengths, amount of dart and cut lines need to be adjusted according to differences in individual back shapes and hip shapes (Momota H. and Makabe H., 1998). Poorly fitting clothing can cause wrinkles, discomfort and they just do not look good.

By using 3D human body data measured by 3D scanner, it is possible to make personalized dummies for use in fitted pattern making. However it is expensive and difficult to find enough storage for physical dummies. Therefore, computerized 3D body models of the human have recently attracted attention in the apparel industry. The development of 3D body model for better fitting clothing is continuing (Peter R .M .J , Peng , Katherine, 1995; Stylios G.K., Han F. and Wan T.R., 2001; Inui S., 2001). Although there are a number of research efforts in the field of 3D body modeling, almost none of them are focused on posture adjustment, they are focused on body mechanics. Also they are not enough to correspond to individual variations in the body depth of the human body.

Another of obstacle in pattern making is variation in individual depth of the body. Even though cross sectional shapes may have similar perimeters, some people are wide from side to side and narrow front to back, while others are thin from side to side and thicker in depth. This affects posture and individual depth as defined above. Therefore it is essential to take into account individual body perimeter, posture and depth of body using computerized 3D body models for pattern making in order to solve such fitting problems.

This paper describes the development of an interactive body model that can be adjusted to match individual body perimeter and posture including depth for the purpose of computerized pattern making . Using three-dimensional body shape data, we extract the information of perimeter, length and angle of back and hip shape to establish individual body perimeter, posture and depth. Also this paper is based on our previously published paper, which describes the development of an interactive body model whose perimeter and length can be altered to model individual body shapes. Because methods for altering perimeter, length, and scaling method are described in our earlier paper, they have been omitted from this paper (Cho Y.S., Okada N., Park H.J., Takatera M., Inui S. and Shimizu Y., 2005).

The process of body modeling is divided into two parts. One part is for controlling the vertical lengths, perimeters and depth, and the other part is for controlling individual postures.

\section{Process of posture body modelling}

1) Extraction of key posture points

To represent the individual body posture, it is essential that ten key points. They are extracted from the side view of the body silhouette. Figure 1 shows the necessary points and positions for constructing different postures. The ten points include seven position control points; neck point, bust point, waist point, stomach point, hip point, crotch point of body (P1 P6) and new waist point 
(W2), and three tangent point from back neck point, waist on back and on hip (S1 S3). Especially points P1, S1, S2, S3 and W2 are added for establishing of body posture. P1 is extracted by line with minimum $+Z$ value above crotch line. $W 2$ is extracted by line with minimum $-Z$ value between waist and hip line.

2) Altering tangent angles of the back and hip parts

Tangent angle of the neck point, waist point and the middle point of stomach and hip $(A, B, C)$ can be altered depending on the individual posture shapes. A and $B$ is for controlling back shape, $C$ is for controlling hip shape.

3) Adjustment of coordinate points

Even though certain body shapes may have the same tangent angles $(A, B, C)$, the body shapes can still have different from coordinate points (P1, P5, P6). So the neck point, hip point and the crotch point of the body model can be moved independently depending on given body shape.

4) Linking the points by spline curves

In the upper part of the body, four points (P1, S1, P2, P3) are linked by spline curves. The cross sectional lines can be altered al ong these spline curves very smoothly. Using eight points (P1, S1, P2, P3, P4, S3, P5, P6) are described with spline curves in the button part of the body.

\section{5) Controlling section lengths}

Control over length from neck to bust $(\mathrm{H} 1)$, bust to waist $(\mathrm{H} 2)$, waist to hip $(\mathrm{H} 3)$ and hip to crotch (H4) allows construction of a more exact body model.

Take in Figure 1

6) Modeling various hip types

By controlling $C$ angle, we can model various hip types, such as flat hip shape and protruding hip shape. Even though two bodies may have the same C angle, their curves between W2 and S3 can still be different as shown in Figure 2 (b). Therefore in this paper, hip shapes can be divided into three types $(T 1, T 2, T 3)$ by the ratio of angles $K h s$ and $K s w 2$. Also we extracted new point Sw2. The Sw2 is middle point from waist to stomach (Figure 2(a)). The $K h s$ is the angle at the tangent point of S3 from W2 (Figure 2(a)). The Ksw2 is the angel at the tangent point of Sw2 from W2 (Figure 2(b)). SK is the ratio of $K s w 2$ to $K h s$ as described in equation (1). U sing 3D measured data of twenty people, we divided them into T1, T2 and T3 types. Therefore we obtained average of $S K$ is valued $0.6,0.85$ and 1.05 , respectively.

To adjust individual hip types, $K_{s w 2}$ is calculated by multiplying $C$ angle by $S K$. The $K_{s w 2}{ }^{\prime}$ is converted to the new $Z$ value which replaces the existing $Z$ value (Bzsw2). As moving Bzsw2, T1, T2 and T3 type is provided. Calculation method of $K_{s w 2}{ }^{\prime}$ and $B z_{s w 2}$ is described in equation (2 4). Therefore it is possible to model for three different hip types despite having same $C$ angel using this method. 
Take in Figure 2

$$
\begin{aligned}
& S K_{i}=\frac{1}{m_{i}} \sum_{0}^{m \in T_{i}} \frac{K_{s w 2}}{K_{h s}} \quad(\mathrm{i}=1,2,3) \\
& K_{s w 2}{ }^{\prime}=C \times S K \\
& a=\frac{1}{\tan \left(K_{s w 2}{ }^{\prime}\right)} \\
& B z_{s w 2}=\frac{1}{a}\left(y_{s w 2}-y_{s 3}\right)+B z_{s 3} \\
& m_{i} \quad: \text { Numer of Ti hip types data }(i=1,2,3) \\
& \text { C : Tangent angle at s3 } \\
& \text { a : Slope of tangent angle } \\
& B z_{s w 2} \quad: Z \text { value at sw2 } \\
& \mathrm{Bz}_{\mathrm{s} 3} \quad: \mathrm{Z} \text { value at } \mathrm{s3} \\
& y_{s w 2} \quad: Y \text { value at sw2 } \\
& y_{s 3} \quad: Y \text { value at s3 }
\end{aligned}
$$

\section{Controlling depth of body model}

For controlling the depth of body, we calculated diameters from side to side (width) and from front to back (depth) of cross sectional lines of bust, waist and hip (Figure 3(a)). The widths are expressed as $X$ coordinates and the depth as $Z$ coordinates. Scaling ratio of depth ( $D s c / X, D s c / Z$ ) is described as the diameter of the cross-sectional line of the basic model (D[bust], D[waist], D[hip]) and the diameter of the cross-sectional line of the modified body model (SD[bust], SD[waist], SD[hip ), as described in equation $(5,6)$. We control diameter $(\mathrm{cm})$ from front to back of cross sectional lines as depth.

To smooth the body shape, other lines in the whole body are divided into five sections for controlling depth as shown in Figure 3 (b). The formulae of scaling ratio for depth are described as follows in equation (7 13). 


$$
\begin{aligned}
& \operatorname{Dsclz}[g]=\frac{S D[g]}{D[g]} \\
& \operatorname{Dsclx}[g]=1+\left(1-\frac{S D[g]}{D[g]}\right) \\
& \text { - bust } \leq B T \leq \text { top } \\
& \text { Dsclz[BT ] = Dsclz [bust ] } \\
& \operatorname{Dsclx}[B T]=\operatorname{Dsclx}[\text { bust }] \\
& \text { - waist } \leq W B \leq \text { bust } \\
& D s c l z[W B]=\frac{D s c l z[\text { bust }]+D s c l z[\text { waist }]}{N_{w b}}(h-\text { bust })+D s c l z \text { [bust ] } \\
& \operatorname{Dsclx}[W B]=\frac{D s c l x[\text { bust }]+D s c l x[\text { waist }]}{N_{w b}}(h-\text { bust })+D s c l x[\text { bust }] \\
& \text { - stomach } \leq S W \leq \text { waist } \\
& D s c l z[S W]=\frac{D s c l z[\text { waist }]+D s c l z[\text { hip }]}{N_{s w}}(h-\text { waist })+D s c l z \text { [waist ] } \\
& \operatorname{Dsclx}[S W]=\frac{D s c l x[\text { waist }]+D s c l x[\text { hip }]}{N_{s w}}(h-\text { waist })+D s c l x[\text { waist }] \\
& \text { - hip } \leq H S \leq \text { stomach } \\
& \text { Dsclz [HS ] = Dsclz [ hip ] } \\
& \text { Dsclx [HS ] = Dsclx [hip ] } \\
& \text { - thigh } \leq T H \leq \text { hip } \\
& \operatorname{Dsclz}[\mathrm{TH}]=\frac{\operatorname{Dsclz}[\text { hip }]+1}{N_{t h}}(h-\text { hip })+\operatorname{Dsclz}[\text { hip }] \\
& \operatorname{Dsclx}[\mathrm{TH}]=\frac{\operatorname{Dsclx}[\text { hip }]+1}{N_{t h}}(h-\text { hip })+\operatorname{Dsclx}[\text { hip }] \\
& \text { vertexd }[h] . x=D \text { Dsll } \times \text { vertexs1[h].x } \\
& \text { vertexd }[h] . z=D \text { sclz } \times \text { vertexs } 1[h] . z
\end{aligned}
$$

$\begin{array}{ll}\operatorname{Dsclz}[g] & : \text { Scaling ratio of depth in } Z \text { coordinates } \\ \operatorname{Dsclx}[g] & : \text { Scaling ratio of depth in } X \text { coordinates } \\ \text { vertexd }[h] . x, \text { vertexd }[h] . z & : \text { Coordinates of point after depth control }(\mathrm{x}, \mathrm{z}) \\ \operatorname{vertexs} 1[h] . x \text {, vertexs } 1[h] . z & : \text { Coordinates of point after scaling }(\mathrm{x}, \mathrm{z}) \\ g & : \text { Line number for control depth } \\ h & : \text { Number of cross sectional lines } \\ N_{p} & : \text { Number of lines included in each section } \\ n & : \text { Number of points in each line }\end{array}$




\section{Result}

\section{Representation of individual back shapes and hip types}

Figure 4 (a) shows the results of altering A, B angle for modelling back shape such as flat shape, average shape and stoop shape. Figure 4 (b) shows different hip shapes using controlled $C$ angle. Figure 4 (c) also shows various hip shapes with different $K_{s w 2}$ 'angle, and sharing the same $C$ angle. The various body shapes in Figure 4 show that our presented body model is able to accurately simulate individual back and hip shapes.

\section{Take in Figure 4}

Results of controlling depth of body

Examples of modelling bust, waist and hips that have the same perimeter but different depth is shown in Figures 5, 6 and 7 .

By controlling the front to back depth of certain body parts independently, it is possible to model bodies having different front to back depth in upper and lower halves of the body.

\section{Take in Figure 5}

Take in Figure 6

Take in Figure 7

\section{Comparison of real human body shapes with virtual body model shapes}

In order to confirm whether our virtual model can be adequately substituted for real human body, we compared the side silhouette line of scanned real body shape. It is necessary to be able to modify posture and front to back depth of the virtual body model.

The models are superimposed in Figures 8 and 9 to allow easy comparison. Figure 8 (a) show models with adjusted perimeter but non-adjusted posture superimposed over a real body shape. Figure 8 (b) show models with adjusted perimeter and posture superimposed over a real body shape. Figure 9 (a) show models with adjusted perimeter but non-adjusted posture superimposed over a real body shape. Figure 9 (b) show the adjusted perimeter and posture but non-adjusted depth of bust superimposed over a real body shape. Figure. 9 (c) show models with adjusted posture, perimeter and depth of bust superimposed over real body shape.

We can see an almost perfect match between the fully adjusted model shapes and real body shapes.

\section{Take in Figure 8}

Take in Figure 9

\section{Development of primary dialog and posture dialog for the body model}

The software interface is divided into two parts, being the primary dialog and the posture dialog. Perimeter, length and front to back depth controls are positioned in the primary dialog. Back and hip shape controls are positioned in the posture dialog. 
In the primary dialog, the interface has 7 panels to view and adjust the interactive body model. They show a cross section of the model itself ( $g$ ), the basic adjustment controls (b), size and length information (d) and controls for adjusting the individual depths of bust, waist and hips (c). Different cross sectional views can be selected in panel (f). Panel (a) shows the model itself, and reflects adjustments. Button (e) brings up the posture dialog.

In the posture dialog, panel (i) is provided to select hip types. Panel (j) is used to adjust angles for the shape of the back, and the postural positions of the back and hips. Panel (h) shows the posture model itself, and reflects adjustments.

Take in Figure 10

\section{Conclusion}

This paper describes the development of an interactive body model that can be altered to match individual body perimeters and postures, including depths, for the purpose of computerized pattern making . Construction of posture adjustable body model requires the extraction of necessary points, adjustment of coordinate points, linking of points by spline curves, controlling of section lengths and selectability of various hip types. Moreover, construction of depth adjustable body model requires the calculation of scaling ratio from length of front to back depth of body.

Silhouette comparison between the fully adjusted virtual body model and real body shapes shows an almost perfect match. These results support the adaptability and potential usefulness of the posture and depth adjustable body model.

Also we developed a primary dialog for altering perimeter, length and depth, and a posture dialog for controlling back and hip shapes. It can be helpful to adjust individual body shape using these dialogs.

By making fine adjustments to the posture and the depth, it is possible to make patterns which result in clothing that not only fits well, but also exhibits other desirable properties. These other desirable properties, such as drape style, dart amount and optimum use, are viewable on screen before any fabric has even been cut. This system could, therefore, be seen as a major step forward in pattern making.

\section{Acknowledgement}

This research was supported by the Ministry of Education, Science, Sports and Culture, Grant-in-Aid for 21st Century COE Program and Scientific Research (C), 16500124, 2005.

\section{References}

Cho Y.S. ,Okada N., Park H.J . ,Takatera M., Inui S. and Shimizu Y. (2005), “An interactive body model for individual pattern making", International Journal of Clothing Science and Technology, Vol 17 No 2, pp. 91-99

Hashizume S. and Nagata M. (1999), "Study on the fittability of clothing size", Sen'i Senihin Shohi 
Kagaku, Vol 40 No 4, pp. 246-254

Inui S. (2001), "A preliminary study of a deformable body model for clothing simulation", International J ournal of Clothing Science and Technology, Vol 13 No 5, pp. 339-350

Masuda T. and Imaoka H. (2002), "Feature examination of curved shapes for tight-fitting patterns of young women by using concentrated gaussian curvature", J . Home Econ. J pn, Vol 53 No 8, pp. 795-804

Matsuyama Y., Yamazaki I., Watanabe K. (1998), "Individual characteristics of dorsal curves as a factor of apparel fit", J. Home Econ. J pn, Vol 49 No 1, pp. 69-76

Momota H. and Makabe H. (1998), "Physical type characteristics of the upper half body of J apanese adult males-Part 1 : Examination by body measurement and development plane Figureures", J ournal of the J apan research association for textile End-Uses, Vol 39 No 6 , pp. 386-391

Momota H. and Makabe H. (1998), "Physical type characteristics of the upper half body of J apanese adult males-Part 2 : Relation between measurement items and basic bodice pattern", J ournal of the J apan research association for textile End-Uses, Vol 39, No 8, pp. 517-526

Peter R . M . J ones, Peng Li, Katherine Brooke-Wavell and Gordon M . West (1995), "Format for human body modeling from 3-D body scanning", International J ournal of Clothing Science and Technology, Vol 7 No 1, pp. 7-16

Stylios G.K., Han F. and Wan T.R. (2001), "A remote on-line 3-D human measurement and reconstruction approach for virtual wearer trials in gl obal retailing", International J ournal of Clothing Science and Technology, Vol 13 No 1, pp. 65-75

Takabu H. and Matsuyama Y. (1987), "Analysis of Side View of Human Body Shape”, J. Home Econ. J pn, Vol 38 No 11, pp. 999-1007

WatanabeY. (1999), "Ordering your cloth to fit yourself", The ournal of the Institute of Electronics, Information and Communication Engineers, Vol 82 No 4, pp. 404-411 


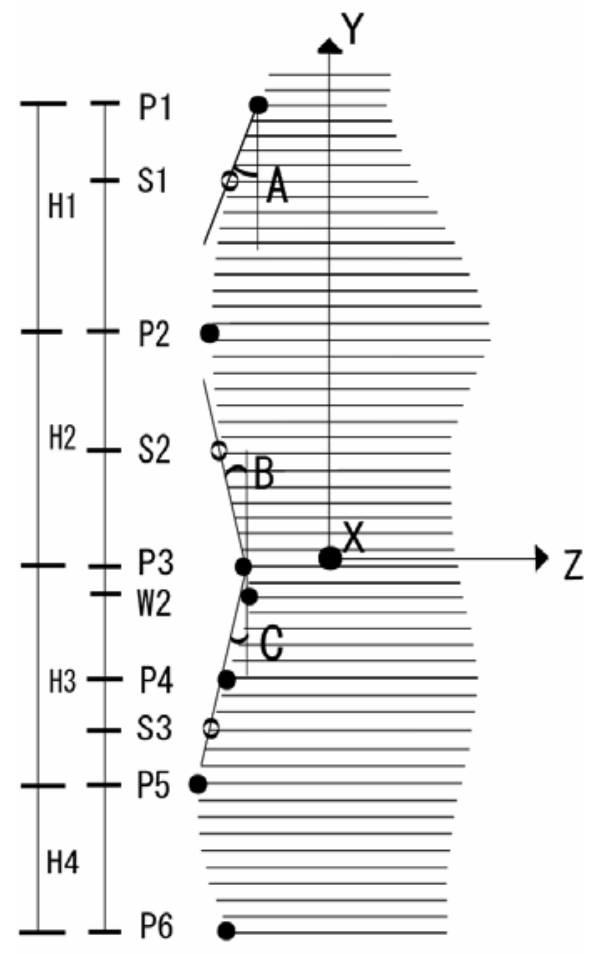

P1 : Back neck point

P2 : Bust point

P3 : Waist point

P4 : Stomach point

P5 : Hip point

P6 : Crotch point

S1 : Tangent point from $\mathrm{P} 1$

S2 : Tangent point from $\mathrm{P} 3$

S3 : Tangent point at middle of P4 and P5

A : Tangent angle at S1 from $\mathrm{P} 1$

$\mathrm{B}$ : Tangent angle at $\mathrm{S} 2$ from $\mathrm{P} 3$

$\mathrm{C}$ : Tangent angle at S3 from $\mathrm{P} 3$

$\mathrm{H} 1$ : Length from neck to bust

$\mathrm{H} 2$ : Length from bust to waist

H3 : Length from waist to hip

H4 : Length from hip to crotch

W2 : Waist point with minimum $-Z$ value between waist and hipline.

Figure 1. Necessary points for constructing individual posture 


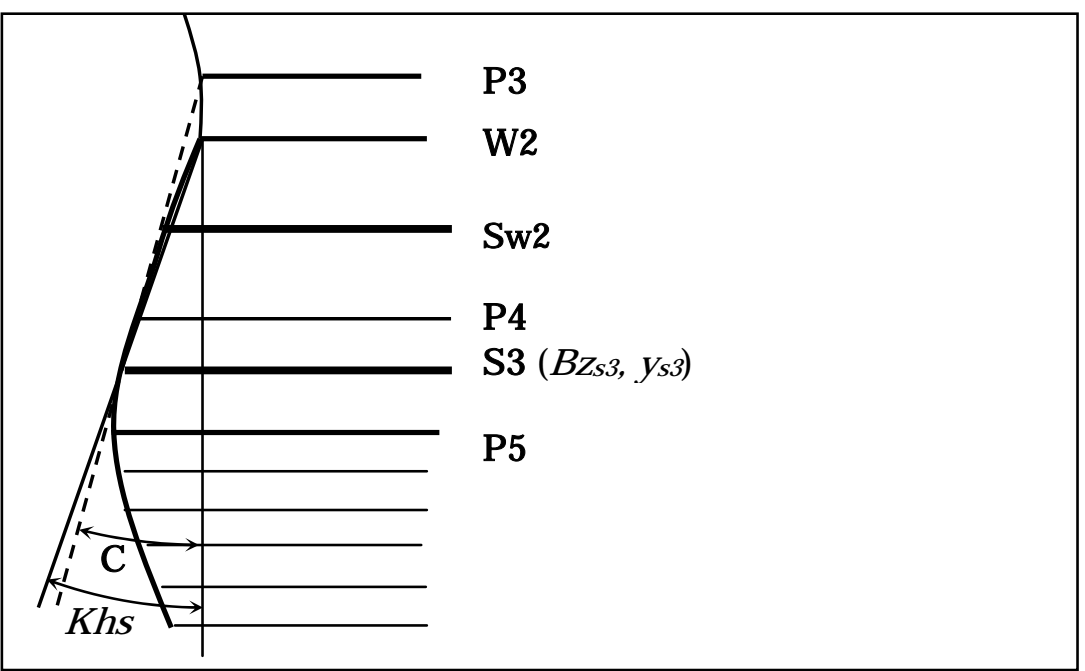

(a)

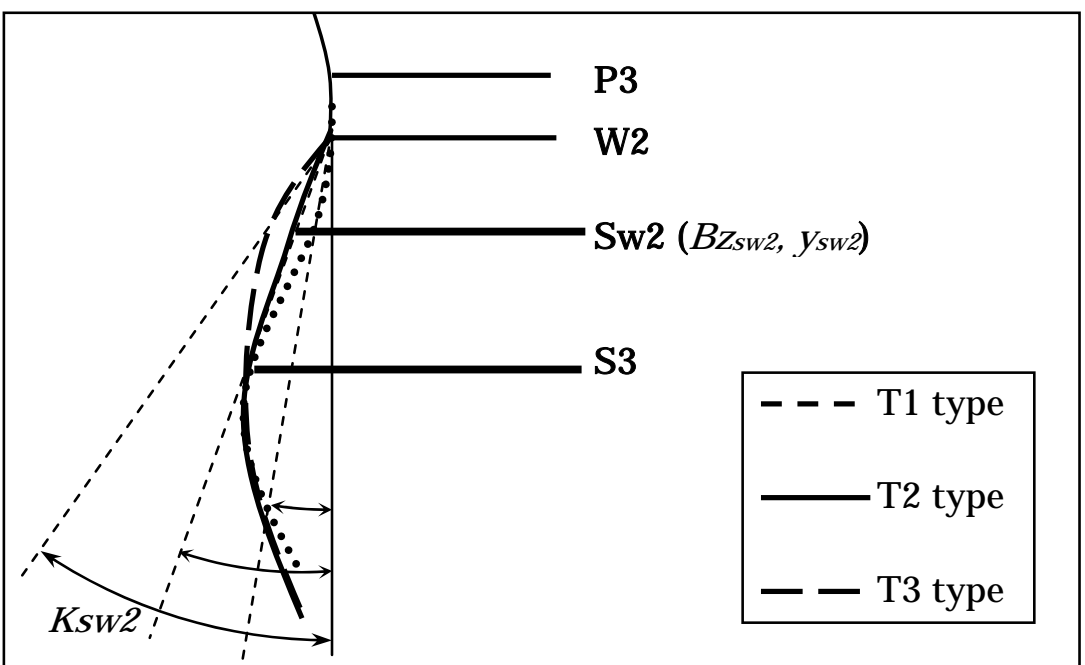

(b)

Figure 2. Necessary points and angles for modeling hip types 


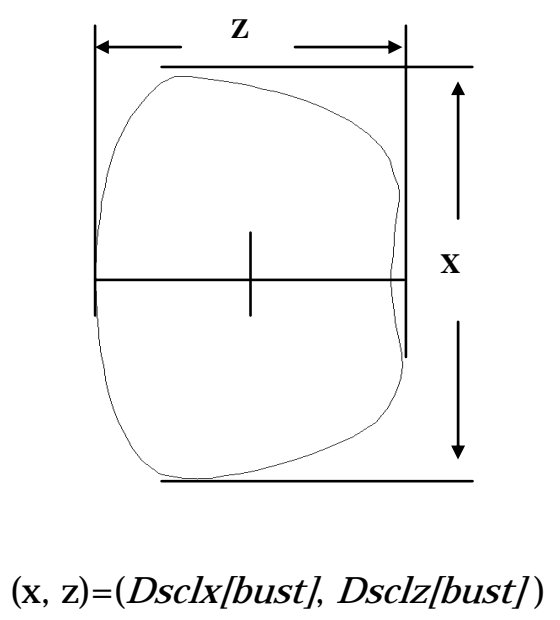

(a)

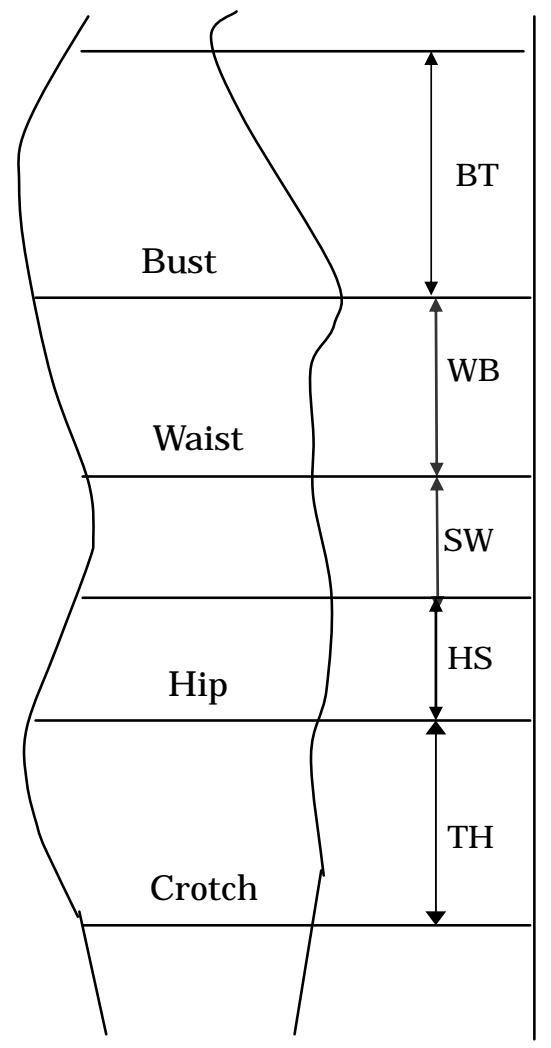

(b)

Figure 3. Information for controlling depth of body 


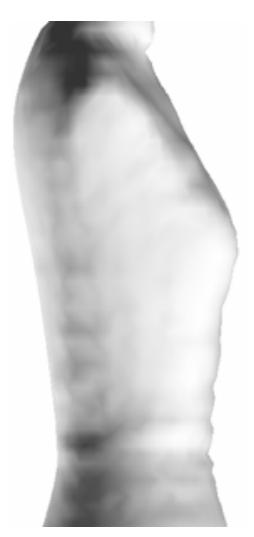

(a) Flat back shape (A:20 $B: 9^{\circ}$ )

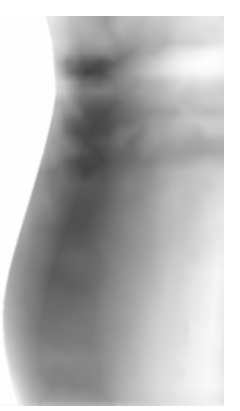

(b) Flat hip shape $\left(\mathrm{C}: 11^{\circ}\right)$

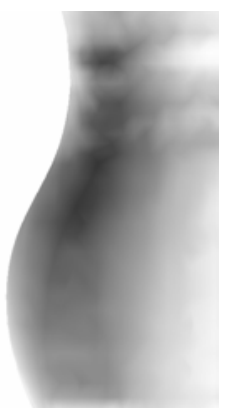

(c) T1 type (C:15० $K_{s w 2^{\prime}: 9^{\circ}}$ )

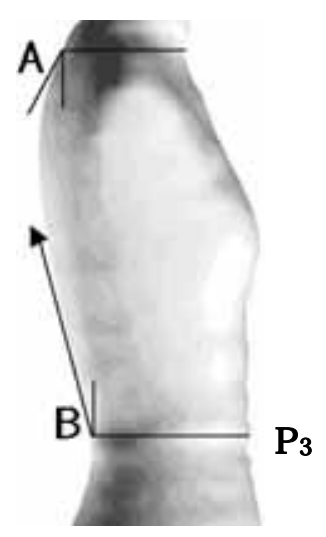

Average back shape $\left(A: 23^{\circ} B: 14^{\circ}\right)$

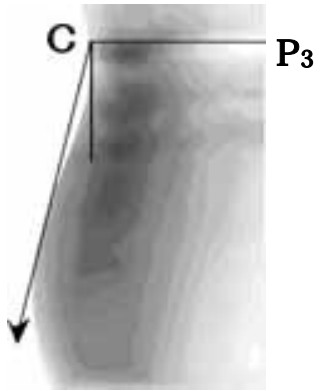

Average hip shape (C:15)

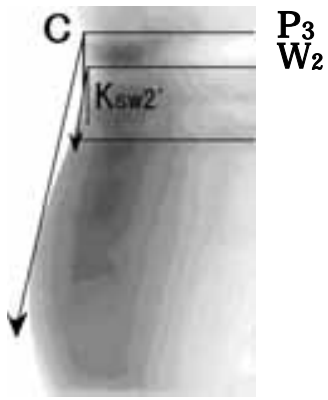

T2 type

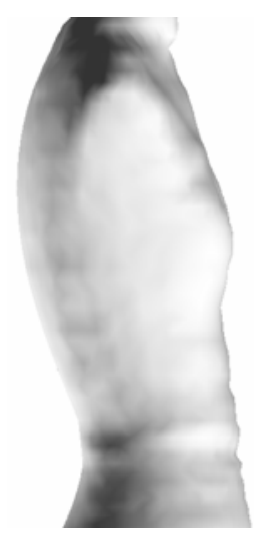

Stoop back shape $\left(A: 26^{\circ} B: 19^{\circ}\right)$

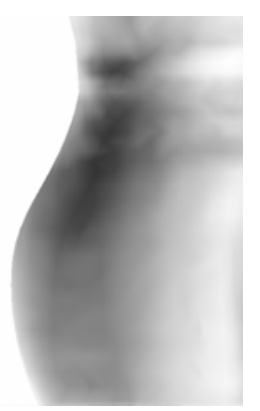

Protruding hip shape $\left(\mathrm{C}: 18^{\circ}\right)$

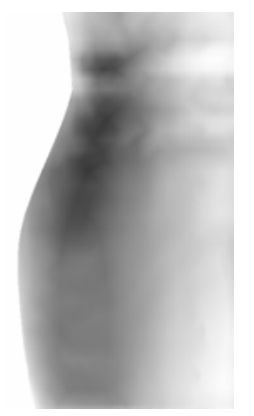

T3 type

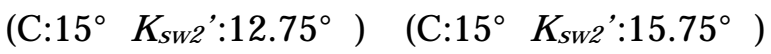

Figure 4. Representations of individual back and hip shapes 


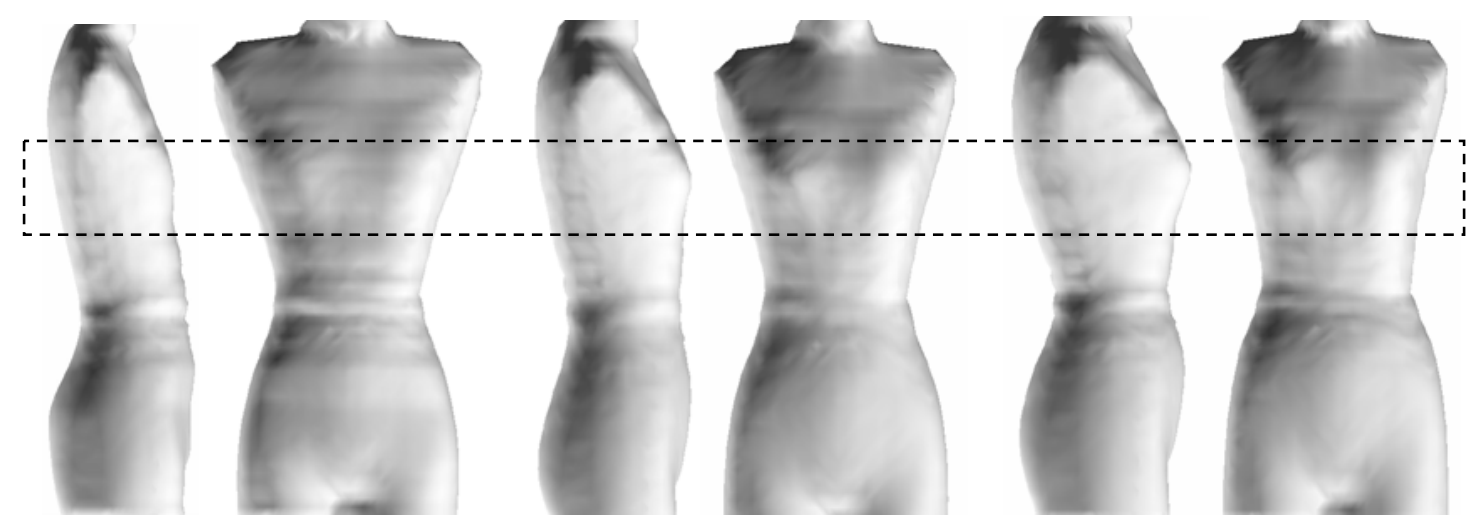

Bust Perimeter $(\mathrm{cm}): \quad 83$

83

83

Bust Depth $(\mathrm{cm}): \quad 18$

21

24

Figure 5. Result of controlling depth of bust

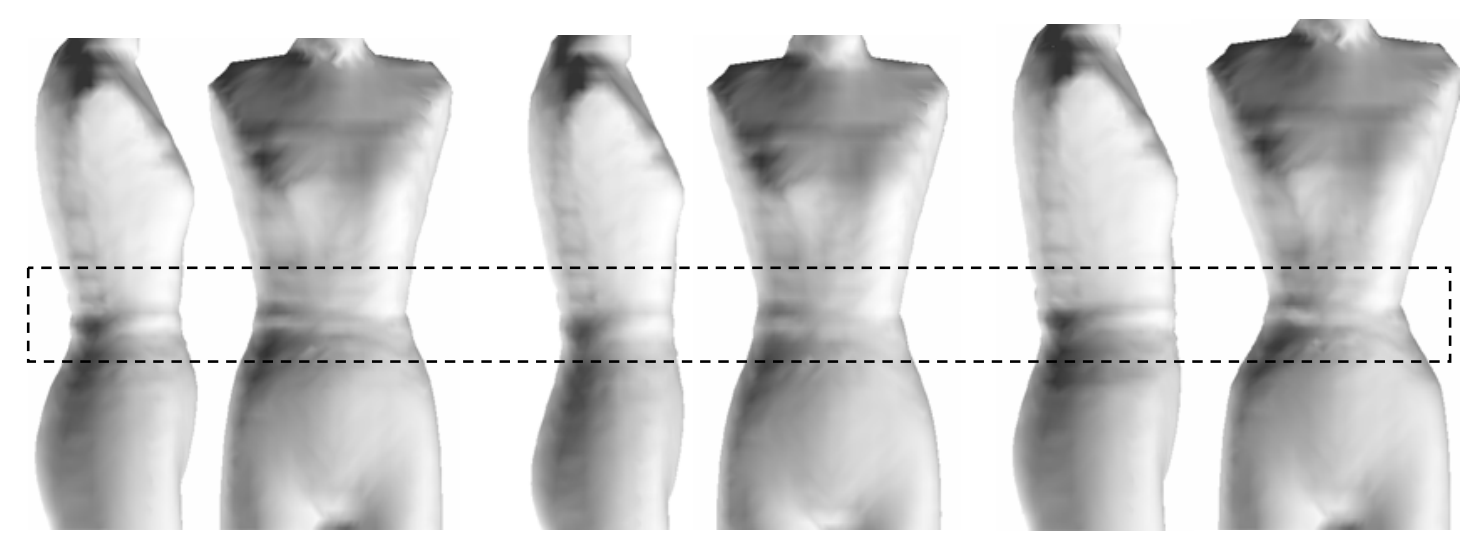

Waist Perimeter (cm): 63

63

Waist Depth (cm):

15

16

Figure 6. Result of controlling depth of waist

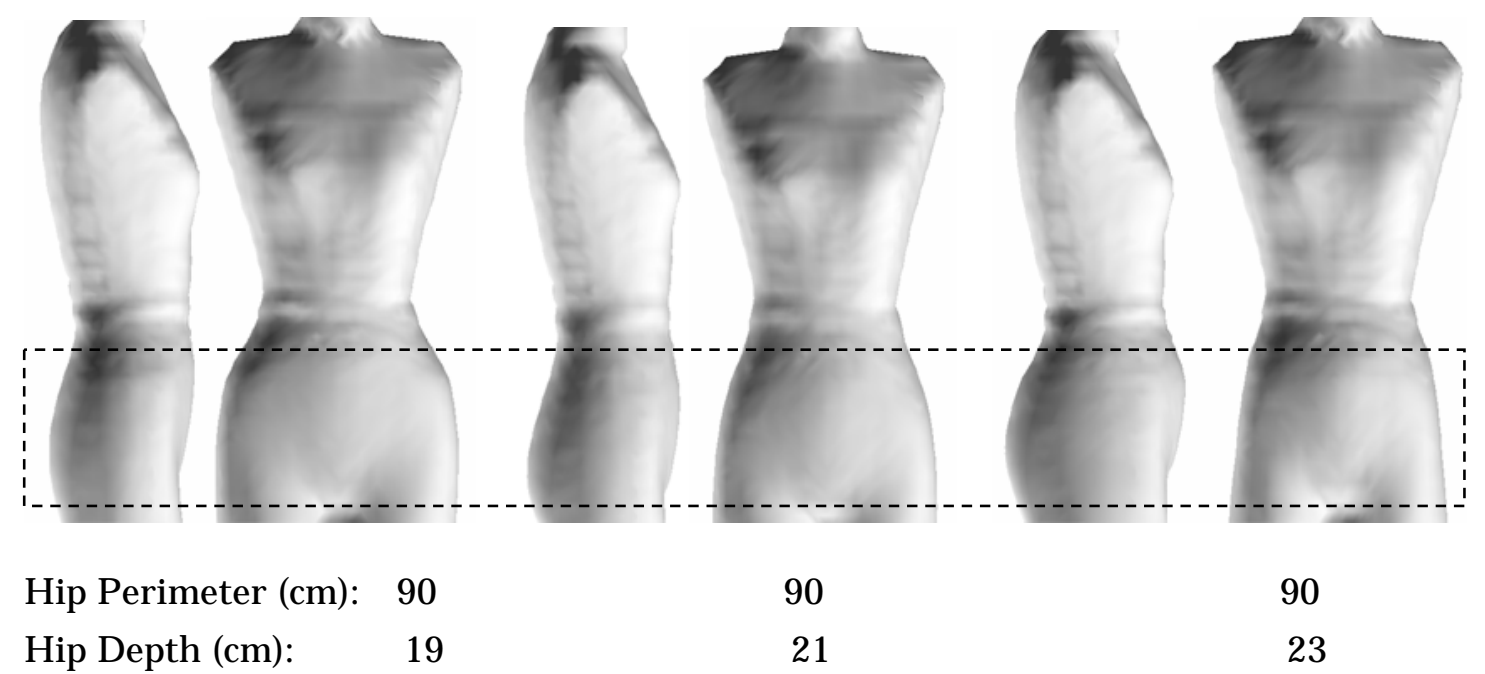

Figure 7. Result of controlling depth of hip 


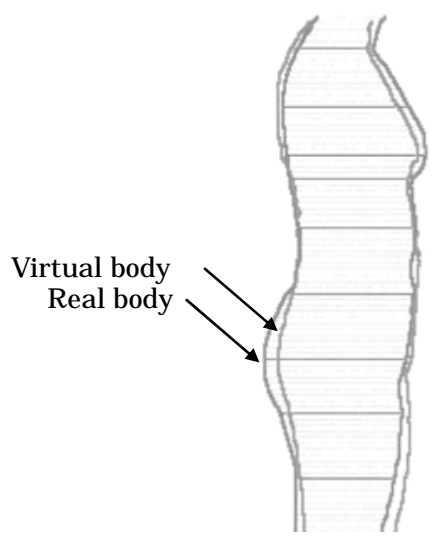

(a)

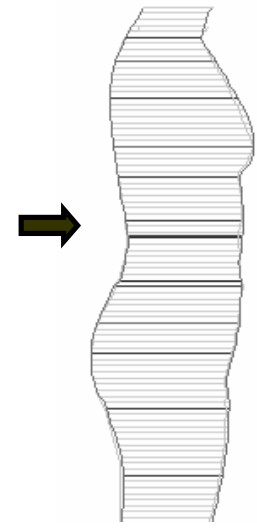

(b)

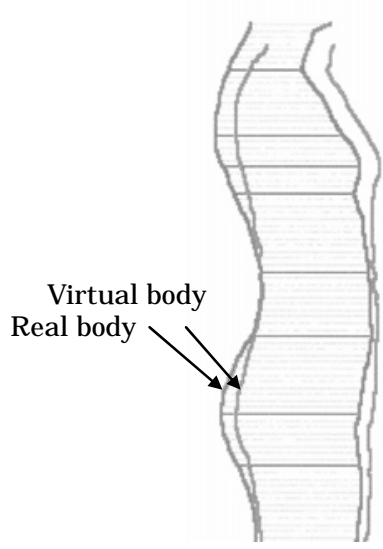

(a)

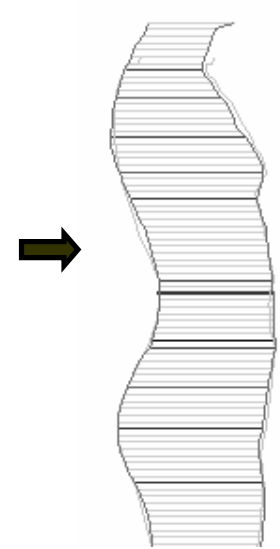

(b)

Figure 8. Result of controlling only perimeter (a)

and controlling perimeter and posture (b)

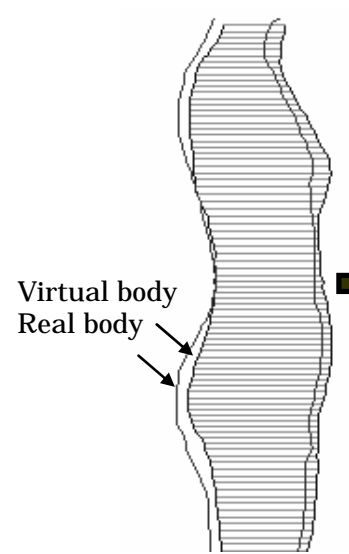

(a)

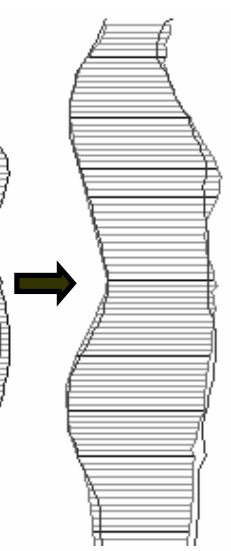

(b)

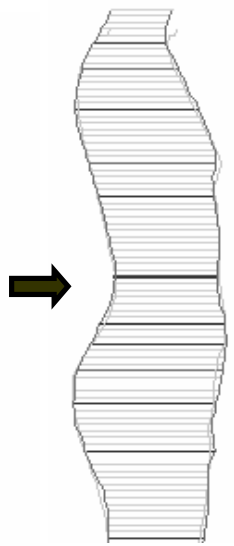

(c)

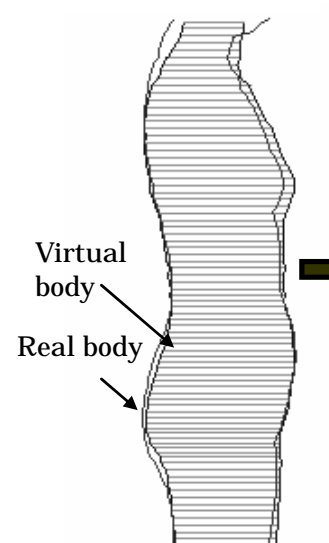

(a)

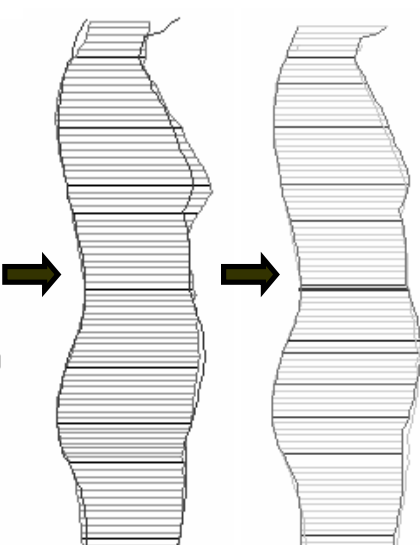

(b)

(c)

Figure 9. Result of controlling only perimeter (a), controlling perimeter and posture (b) and controlling perimeter, posture and depth (c) 
(g)

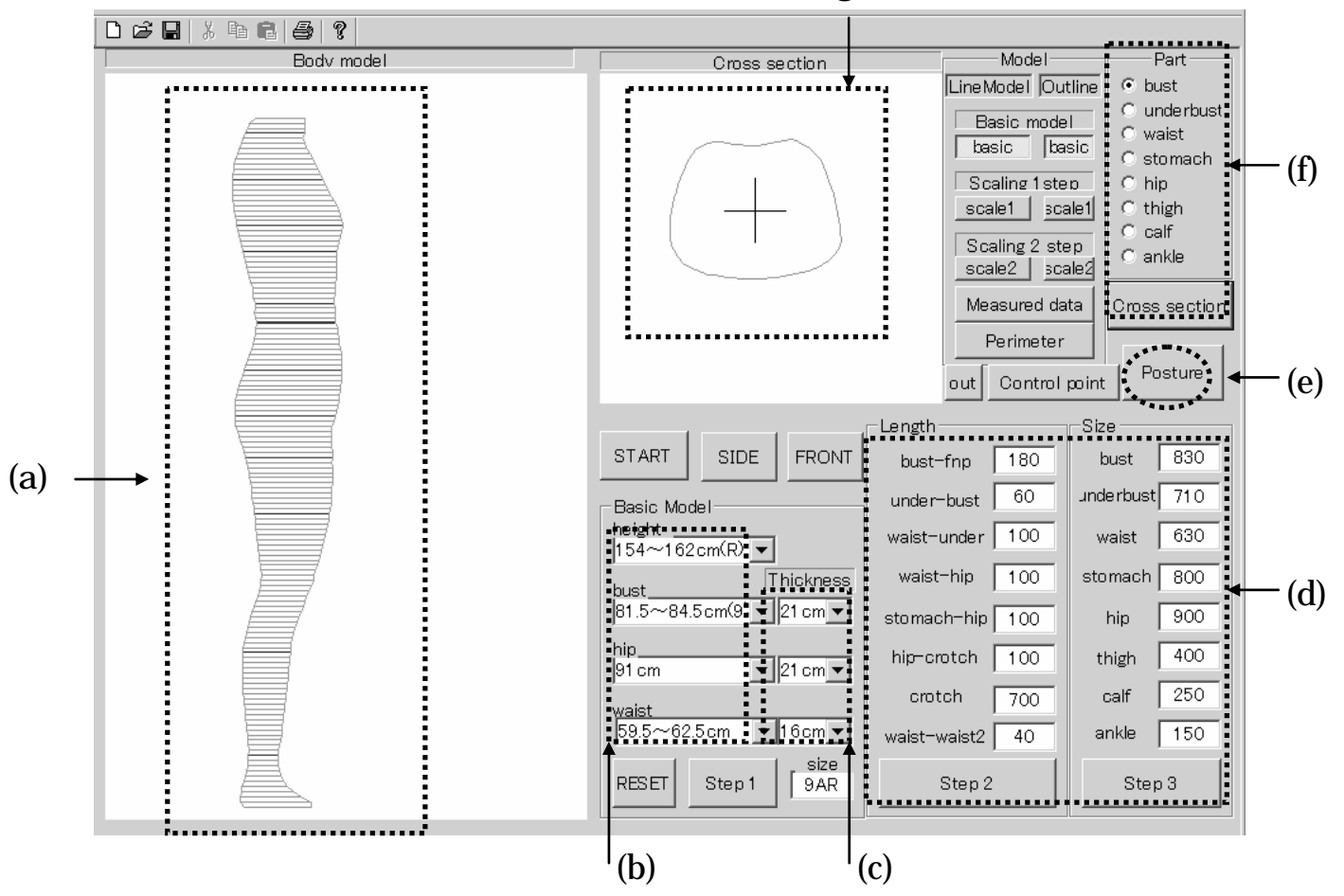

I ) Primary dialog

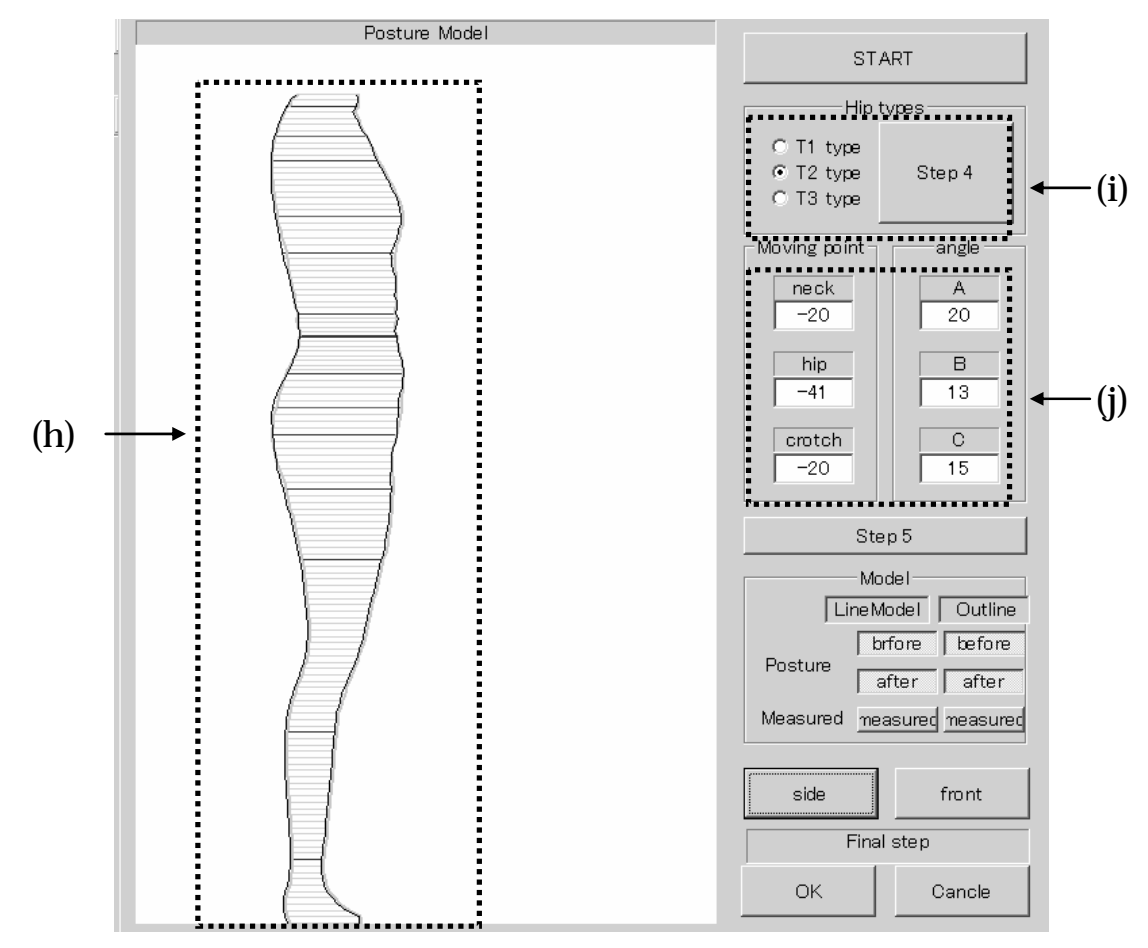

II ) Posture dialog

Figure 10. Primary dialog and posture dialog 